\title{
Mesh Sensitivity Study of Turbine Wheel Burst Analysis using Explicit Dynamics
}

\author{
S.S.Borikar ${ }^{1}$ K.A.Mahajan ${ }^{1}$ G. Hemanth $\operatorname{Kumar}^{2}$ \\ ${ }^{1}$ (Department of Mechanical Engineering, M.E.S College of Engineering, S P Pune University, India) \\ ${ }_{2}^{2}$ (Applied Mechanics, Cummins Turbo Technologies, Pune, India)
}

\begin{abstract}
The containment test in turbocharger is performed to examine the bursted wheel containing capability of the turbine housing. The experimental test demands high investments and is time consuming. Hence, the containment test for turbocharger is simulated using Explicit Dynamics. To facilitate wheel burst, the groove is cut in the back face of the wheel to weaken it. The wheel burst simulated with circumferential groove as the weakening slot, gives an unequal number of fragments even though the analysis model is cyclic symmetric. This gives inappropriate results through containment analysis. One of the major reasons for randomness in fragment size is meshing of the model. This paper presents various meshing concepts for midrange and light duty wheel to obtain the equal number of fragments. The Pugh selection method was employed to select the mesh concept by rating the concepts against different criteria to minimize randomness in fragment size along with maintaining the accuracy of solution. Different mid-range and light duty wheels when evaluated with highest rated mesh concept gave expected burst scenario.
\end{abstract}

Keywords: containment test, explicit dynamics, mesh concepts, pugh matrix, pattern, turbocharger

\section{Introduction}

The turbocharger is sometimes subjected to a very high temperature of exhaust gases and velocity of $950^{\circ} \mathrm{c}$ and $200000 \mathrm{rpm}$ respectively. At such unfavorable over speed service condition, the strength of turbine wheel is decreased due to elevated temperature and internal bonds of the material breaks at high centrifugal forces. Hence, the turbine wheel is weaken and bursted off into fragments. There are two natural failure mode i.e., hub burst and wheel detachment [1]. This bursted off fragments carry a high kinetic energy with them which when hits and penetrates through the housing causes safety issues as the high temperature exhaust gas may enter the bearing housing that provide oil supply pipelines to the bearings. The housing must be strong enough to contain the fragments within itself. Hence, the containment tests are conducted to check the fragments containing capability of the turbine housing. For containment test, the turbine wheel is weaken by cutting a weakening slot in the back face of the wheel and then the wheel is rotated at a speed much higher than the design speed. In one revolution, the wheel is bursted and the containment test is performed. The experimental tests however, costs high and is time consuming. Hence nowadays, the containment tests are simulated using explicit dynamics. The burst simulation necessitates determination of weakening slot dimensions and the material failure criteria. J M Ramamoorthy [2] presented methodology for estimating the wheel burst speed using AWMHS method to determine the material removal dimensions. This estimated burst speed and material removal dimensions for hub burst were used to obtain the damage criteria for the wheel of ductile material. $\mathrm{L}$. Wang [3] published a methodology for optimizing the weakening slot design to burst a turbine wheel through blade detachment at any specified speed for the wheel. However, the simulation was performed for angular groove using the tetrahedral meshing. Johnson and Cook [4] introduced a five parameter empirical model for materials subjected to large strain, high strain rates and high temperature, used for predicting the behavior of material during extremely dynamic events. Xuan Hai-jun [5] used nonlinear finite element method to study the impact process in containment test with single released blade and has discussed the meshing guidelines for the dynamic analysis. The burst simulation for cyclic symmetry turbine wheel model with circumferential groove gives unequal number of fragments after burst. This can be due to mesh or mass scaling effect. The randomness in the sizes of the fragments after wheel burst may cause under-prediction or over-prediction of the fragments containing capability of the turbine housing. Hence, the wheel must burst with equal fragment size. This paper presents various meshing concepts for midrange and light duty wheel with circumferential groove, to obtain the equal number of fragments after burst. The concepts were then rated for various criteria using a Pugh matrix method for concept selection. The highest rated concept was then evaluated for different light duty and midrange wheels and was found to give equal number of fragments with accuracy. 


\section{Key Concepts In Explicit Dynamics}

The Ansys Explicit Dynamics are suitable to capture the physics of short-duration events involving a very high material deformation. Certain concepts like stability of solution and mass scaling affects the runtime in explicit dynamics as this concepts depends on the mesh size.

\section{A. Stability}

The accuracy and the stability of the solution in explicit problems depends on the time steps and are conditionally stable. The coarse mesh may not predict the accurate results as at large time steps the solution is not stable. The error (between the exact and approximate values) goes on increasing and solution diverges from exact solution, producing ill-condition and hence affecting the accuracy. Whereas the fine mesh with very small mesh sizes may increase the run time for the solution by decreasing the time step. Hence, the time step in Explicit Dynamics is determined by solver using Courant-Fredrick-Levy condition (CFL condition) according to which the time step for the solution is equal to the time taken by the stress or shock waves to propagate through the smallest element in the model. Hence, the time step is controlled by the smallest element in the model. Therefore, the mesh size must be decided with proper scrutiny that would give accurate and stable results in less runtime.

\section{B. Mass Scaling}

Results of nonlinear numerical simulation are highly sensitive to the mesh of finite element [5]. While coarse meshing the complex geometries with small features is impractical, fine meshing guarantees accuracy and stability but with high run time. As the smallest element in the model controls the run time for solution, artificial scaling of density of this small elements increases the time step. This procedure of adding the nonphysical mass to the smallest element in the model is called as mass scaling. However, the mass scaling needs to be used judiciously because if it contributes to excess of overall mass addition or to the critical regions, will affect the accuracy of solution giving unrealistic results. In burst simulation of blade detachment, if excess of mass is added to the blades it may cause the wheel to burst at speed lower than the target burst speed due to increase in the centrifugal forces which otherwise would not have bursted without mass scaling.

\section{Numerical Simulation}

The experimental test for containment test requires the trial-and-error method to obtain the groove dimension for bursting the wheel at the target burst speed. Testing inevitably costs a considerable amount of money and takes time [3]. Hence, the burst simulation for containment analysis is performed using Explicit Dynamics.

\subsection{Assumption}

In the FEA models, it is assumed that [3]

1. Aerodynamics load has no effect on wheel failure.

2. The turbine wheel have uniform density and precise geometry.

3. Materials are isotropic, homogeneous and defect-free.

4. Material failure is independent with respect to the types of loadings, strain rate and temperature.

\subsection{Material Model}

The ductile material of turbine wheel undergoes a dynamic failure at high velocity and temperatures, involving a very high strain rates. Johnson and Cook [4] presented a constitutive model that can be used for a dynamic processes and the basic form of model is readily adaptable to most computer codes since it uses variables which are available in the codes. Hence, Johnson-Cook model was employed as it takes into account high strain rate sensitivity, large deformation, and material softening due to adiabatic heating and damage, so it is quite well suited for turbine wheel burst simulation [6]. The yield stress is defined as:

$$
\mathrm{Y}=\left[\mathrm{A}+\mathrm{B}\left(\varepsilon_{\mathrm{p}}\right)^{\mathrm{n}}\right]\left[1+\mathrm{C} \ln \left(\varepsilon_{\mathrm{p}} *\right)\right]\left[1-\left(\mathrm{T}_{\mathrm{H}}\right)^{\mathrm{m}}\right]
$$

The five material constants are $\mathrm{A}, \mathrm{B}, \mathrm{C}, \mathrm{n}$ and $\mathrm{m}$ where, $\mathrm{A}$ is the initial yield strength, $\mathrm{B}$ is the work hardening constant, $\mathrm{n}$ is the work hardening exponent, $\mathrm{C}$ is the strain rate constant, and $\mathrm{m}$ is thermal softening exponent, $\varepsilon_{\mathrm{p}}$ is the effective plastic strain, $\varepsilon_{\mathrm{p}} *$ is non-dimensional plastic strain rate; $\mathrm{T}_{\mathrm{H}}$ is the homologous temperature, which is defined by

$$
\mathrm{T}_{\mathrm{H}}=\frac{\mathrm{T}-\mathrm{T}_{\text {room }}}{\mathrm{T}_{\text {melt }}-\mathrm{T}_{\text {room }}}
$$


The first set of brackets in Eq.1, gives the stress as a function of strain. The second and third sets of brackets represent the effects of strain rate hardening and temperature softening [4].

The effective plastic strain failure criterion is used as a failure model in the burst simulation for prediction of damage.

\subsection{Geometrical factors of weakening slot}

The circumferential groove dimensions, as shown in Fig.1, are outer diameter of groove, depth of cut and the groove width. J.M. Ramamoorthy [2] and L. Wang [3] presented the workflow for optimizing the weakening slot depth. The initial dimensions were considered from the similar wheel and the depth of cut was varied till the dimensions for specified target burst speed was obtained determined.
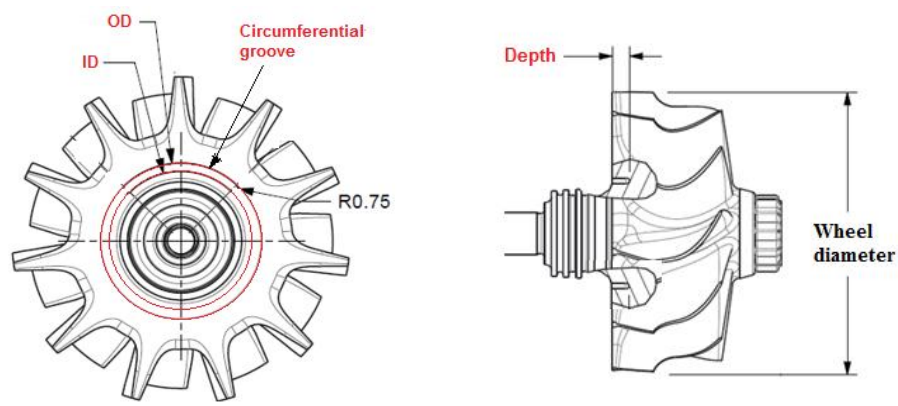

Fig.1. Geometrical factors for circumferential groove

\subsection{Meshing the model}

The simulations done so far for turbine wheel burst with circumferential groove, resulted into an unequal number of fragments. Fig. 2 shows the unequal fragments sizes after blade detachment. The model is symmetric for simulation and circumferential groove is the weakening slot hence, the burst is expected to be with uniform fragments. The variation in the sizes of the fragments after burst results in overestimation or under estimation of the containing capability of the housing wall. This is because the bursted wheel fragments carry kinetic energy which depends on mass of the fragments and variation in the size and mass would affect the kinetic energy and thereafter the impact force. The randomness in the fragment sizes can be due to mesh distribution over the wheel or scaling.

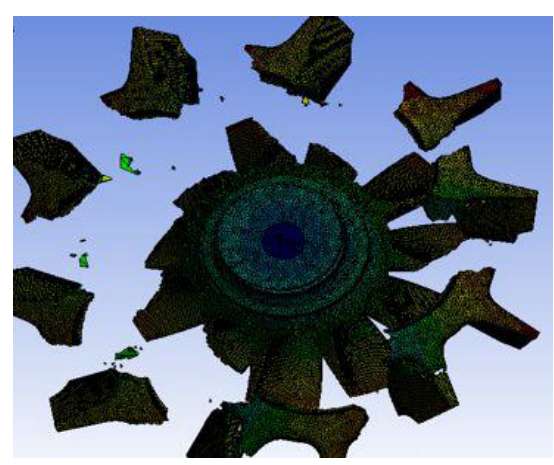
the mass added due to the mass

Fig.2. Present scenario of wheel burst during simulation

One of the major reasons for the randomness in the fragments size after wheel burst is due to improper mesh density over the regions of the turbine wheel. The turbine wheel burst is a dynamic process and hence is simulated using explicit dynamics. The mesh in the explicit dynamics plays an important role in terms that the stress waves in dynamic process propagates throughout the body and continues to move until it is damped. Hence, the stress concentration region in dynamic analysis, unlike static analysis, is not concentrated over one region but moves throughout the body as the stress wave propagates. Therefore, the mesh needs to be uniform throughout the model so that it can predict the results over the entire model as wave propagates. The stability and the runtime for solution depend on the mesh. The mass scaling used to reduce the runtime for solution must be used judiciously, so that the physical mass added is not affecting the accuracy of the solution. The location of 
added mass must be studied as mass added due to mass scaling could be higher, affecting the simulation if added to the critical regions. The tetrahedron mesh is preferred with Explicit Dynamics as it gives uniform meshing over the model.

\subsubsection{Mesh Concepts}

The mesh concepts generated took into account the number of elements and nodes after mesh, mass added due to mass scaling and run time required for the solution. Hence, the model was meshed with different type of mesh algorithm and mesh sizes or by slicing the geometry such that the region which is not of interest can be coarse meshed to reduce the run time. Fig. 3 gives the critical parameter tree for various mesh concepts.

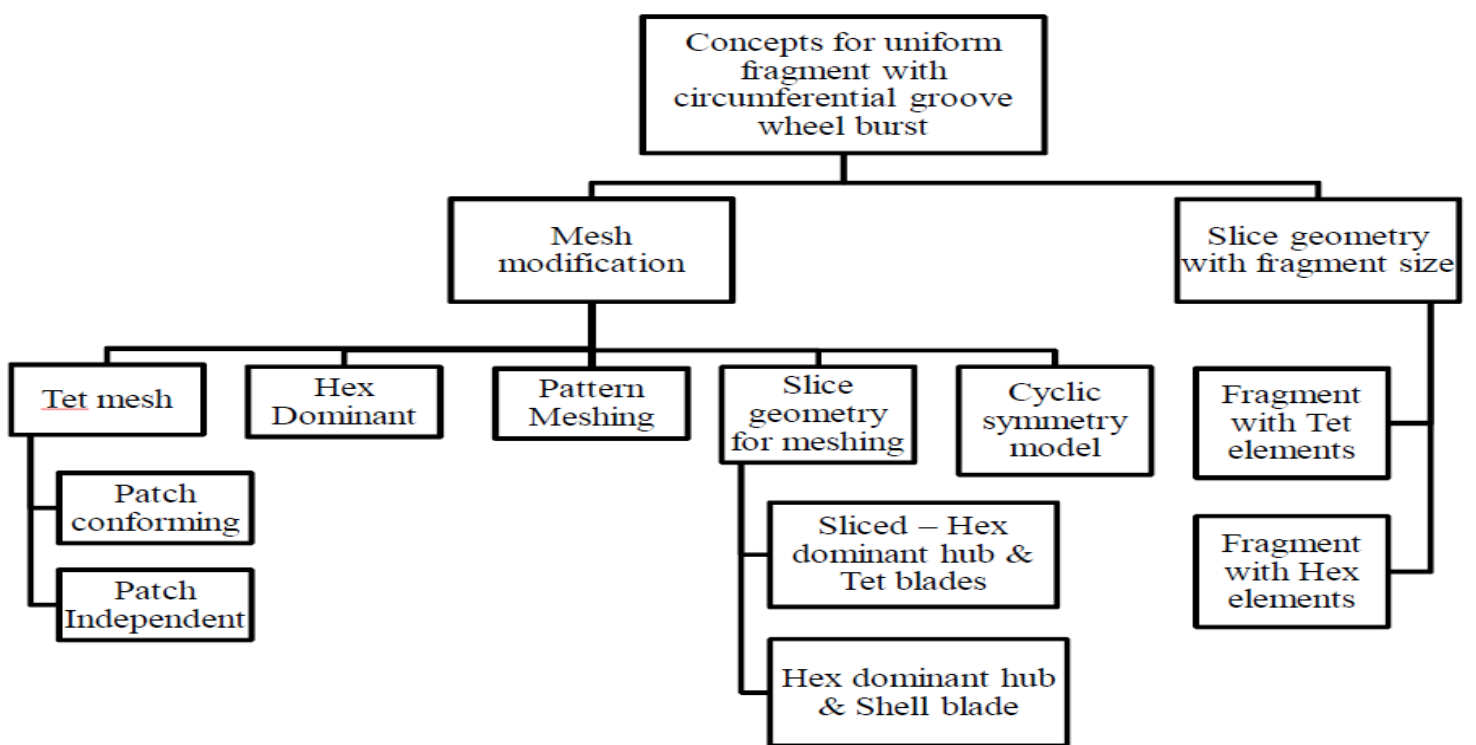

Fig.3. Critical parameter tree for mesh concepts

Tetrahedron mesh creates tet elements and is uniform over the model. Two algorithms are used to create tet elements- Patch independent and Patch conforming. Adaptive and uniform meshing were evaluated with both of this algorithms.

\section{A. Patch Conforming}

The Patch conforming algorithm gives tetrahedron mesh, respecting the features of the model. The faces are meshed first and then the volume of model. However, when meshing highly featured model in explicit dynamics, the runtime for solution and mass added due to mass scaling is high due to smaller element sizes at the features. Fig. 4(a) shows the fine meshing at the features using patch conforming mesh. This mesh concept when evaluated for mid-range wheel with $0.3 \mathrm{~mm}$ mesh size, Fig. 4(b) and light duty wheel with $0.4 \mathrm{~mm}$ mesh size, Fig. 4(c), gave equal fragments with adaptive meshing and required results were not obtained from uniform meshing.

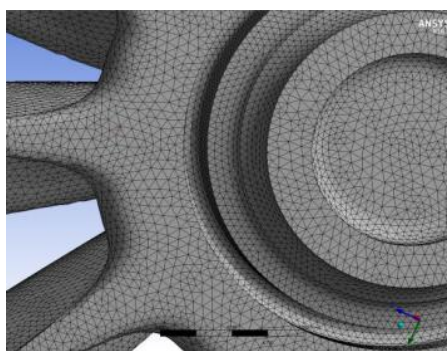

(a)

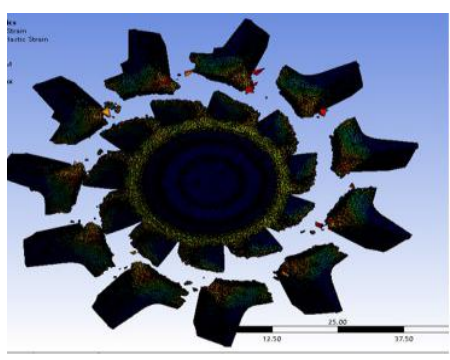

(b)

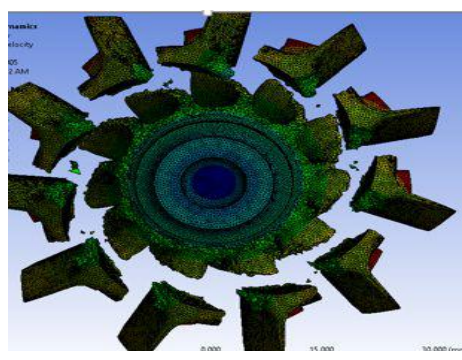

(c)

Fig 4. (a) Patch conforming mesh, (b) MR-0.3 mm mesh size, (c) LD- $0.4 \mathrm{~mm}$ mesh size

\section{B. Patch Independent}

The Patch Independent algorithm meshes the volume first with tetrahedron mesh. Fig. 5(a) shows the model meshed evenly over the surface with patch independent meshing. With adaptive meshing of $0.45 \mathrm{~mm}$ 
mesh size, midrange wheel gave equal number of fragments but light duty wheel gave unequal fragments. Whereas uniform meshing with $0.45 \mathrm{~mm}$ mesh size for midrange wheel as shown in fig. 5 (b), and light duty wheel, Fig. 5(c), gave equal number of fragments.

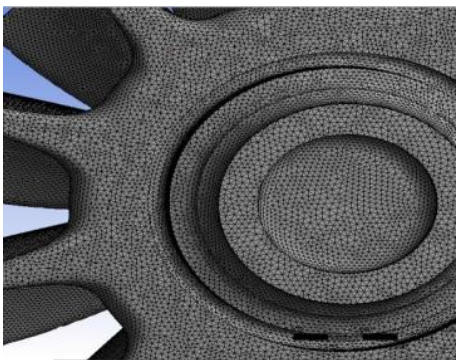

(a)

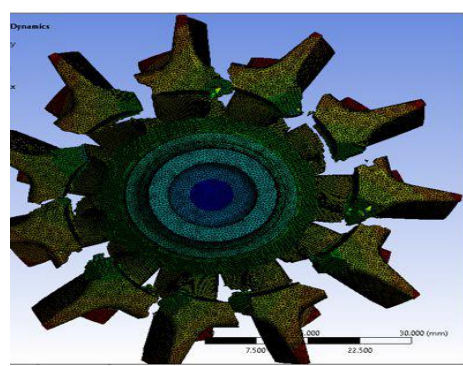

(b)

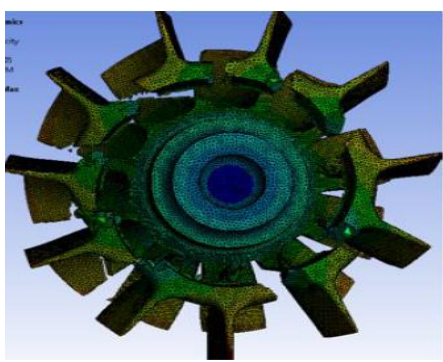

(c)

Fig. 5. (a) Patch independent meshing, (b) $0.45 \mathrm{~mm}$ mesh size, (b) $0.45 \mathrm{~mm}$ mesh size

\section{a. Hex Dominant mesh}

In this mesh, the large portion of wheel is meshed with hex mesh and the pyramids are splitted into tet elements, reducing the element size and hence, increasing the runtime for the solution. This meshing technique is limited for the sweepable body. The wheel was not properly meshed as the wheel body is not sweepable. The mid-range wheel was meshed with $0.45 \mathrm{~mm}$ mesh size and light duty wheel was meshed with $0.25 \mathrm{~mm}$ mesh size for which wheel burst was not observed.

\section{b. Pattern meshing}

In this concept, initially the sector is created. Pattern from the sector is then formed about an axis of rotation as shown in Fig. 6(a). The pattern meshing can be obtained either by form part or instance meshing. The pattern with form part may not necessarily give equal fragments as it considers a single face for the adjacent sectors, sharing the nodes at the faces. Equal fragments were obtained only for light duty wheel using form part. The pattern with instance meshing considers the sectors separately, unlike form part. The sectors are meshed simultaneously in pattern-instance meshing, giving identical number of elements and nodes in each sector and hence the time required for meshing is less. Both the wheels bursted with equal fragments using pattern with instance meshing at $0.5 \mathrm{~mm}$ mesh size, as shown in Fig. 6 (b) and Fig. 6(c).

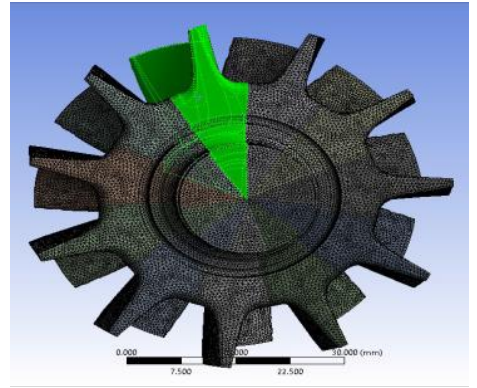

(a)

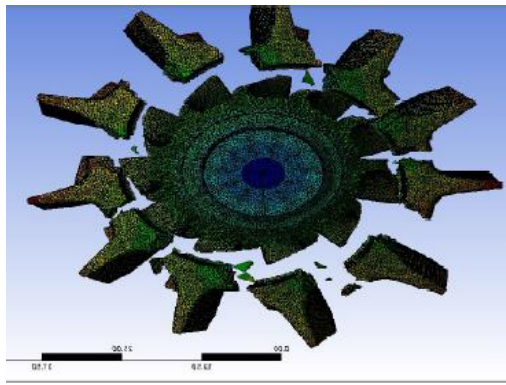

(b)

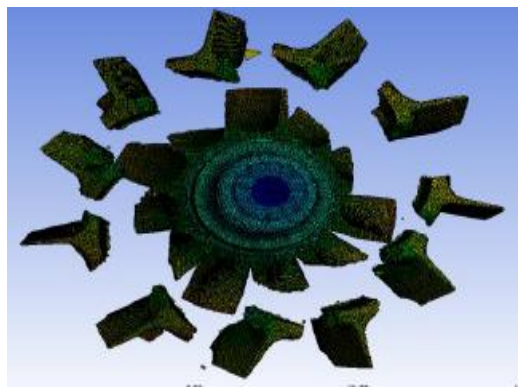

(c)

Fig.6. (a) Pattern-instance meshing, (b) MR- $0.5 \mathrm{~mm}$ mesh size, (c) LD- $0.5 \mathrm{~mm}$ mesh size

\section{c. Sliced geometry for meshing}

The model may contain some region which is not affected in the simulation. Meshing such regions with the same mesh size as domain may increase run time for simulation and excess mass is added to the region which is not area of interest. During wheel detachment, the hub of the turbine wheel is not affected. Hence, the turbine wheel can be sliced to separate hub from the blade so that both this parts can be meshed separately with different mesh sizes. This reduces the run time for solution. The hub and blades can be meshed in two ways as given below:

\section{A. Sliced - Hex dominant hub \& Tet blades}

The hub and blades are sliced and the hub is meshed with the hex dominant mesh and the blades are meshed with the tet elements. The different mesh sizes on the two bodies may not match, increasing time for meshing. The hub of the wheel is meshed with hex dominant mesh of $1 \mathrm{~mm}$ mesh size and blades are meshed with patch 
independent mesh of $0.5 \mathrm{~mm}$ mesh size, Fig. $7($ a). The mid-range wheel bursted with unequal number of fragments shown in fig. 7 (b). The light duty wheel was not meshed properly with this concept.

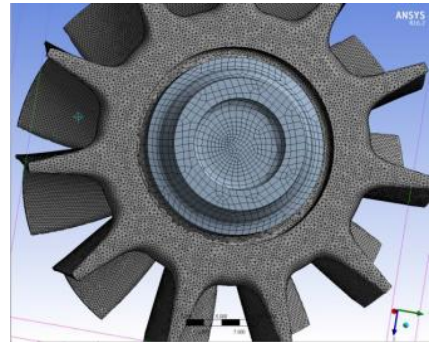

(a)

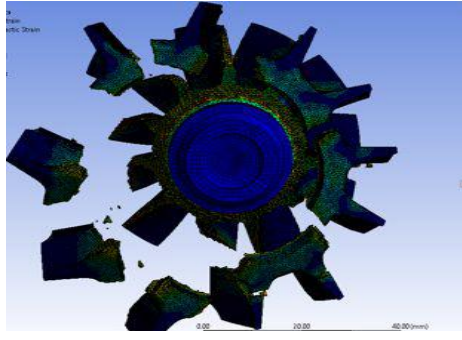

(b)

Fig.7. (a) Sliced body-hex hub and tet blades, (b) hex-1 $\mathrm{mm}$ and tet- $0.5 \mathrm{~mm}$ mesh size

\section{B. Hex dominant hub \& Shell blade}

The blades are meshed with the shell elements and the hub with the hex dominant mesh. The thickness of the blade varies over a length. Hence, it is tedious job to obtain the shell blade. The run time for simulation is less due to less number of elements and nodes but the pre-processing time for creating the shell blade and slicing from the hub is high. This concept was not evaluated as the shell blades were not obtained due to variation in the blade thickness.

\section{d. Cyclic symmetry}

Only a sector from model is considered. This sector is then meshed and analyzed. The result obtained is only for the sector through which the result for complete model can be evaluated. The simulation run time is less as only one sector is analyzed. The burst simulation was performed using Ansys Autodyn that do not support cyclic symmetry, but commands can be inserted for different software that supports the cyclic symmetry commands.

\section{e. Slice geometry with fragment size}

The complete blade is not detached from the hub but, only the outer part of blade through which crack propagates is thrown off after wheel burst. Hence, slicing the wheel through the path of crack propagation gives fine meshing only for fractured blades and other part of wheel can be coarse meshed. The groove is not created in the model as the wheel is sliced according to the path of crack propagation obtained from the burst simulation of complete wheel with the circumferential groove, shown in Fig. 8(a).

\section{A. Fragment with tet elements}

The fragments are meshed with tet elements and the hub is coarse meshed with patch independent mesh of 1 $\mathrm{mm}$ mesh size and blades of fragment size with $0.5 \mathrm{~mm}$ mesh size. The wheel burst was obtained with equal number of fragments, as shown in fig. 8 (b).

\section{B. Fragment with hex elements}

The fragments are to be meshed with hex dominant mesh. However, meshing is difficult even when mesh size is varied.

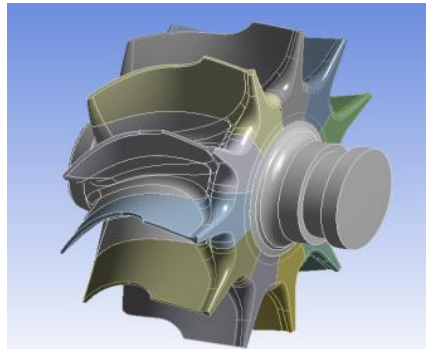

(a)

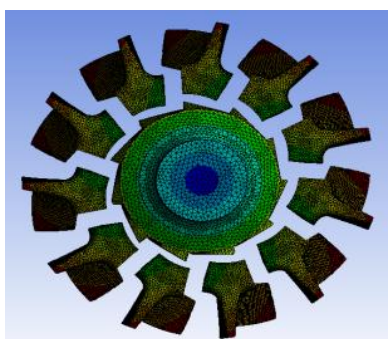

(b)

Fig.8. (a) Sliced geometry with fragment size, (b) MR- $0.5 \mathrm{~mm}$ mesh size

\section{Selection of Mesh Concept}


The selection of the optimum concept from the generated one necessitates the comparison of these concepts among themselves. Pugh matrix method is used for finding the best concept from the generated possible alternatives, giving insights into the strong and weak areas of the concepts. In this method, the selection of concept depends on various criterions that are prioritized by giving weightage to the crucial one. The criteria for mesh concept selection is number of fragments, number of iterations to obtain equal fragments, runtime for solution, mass added due to mass scaling, pre-processing time, development time and complexity involved. The rating for the criteria is given on four point scale. The scores for each concepts are then computed by summation of rating times the weights. The concepts as then ranked in accordance with the scores obtained. The number of fragments and mass scaling effect has maximum weightage as mesh concepts were generated to obtain the equal number of fragments without affecting accuracy of the solution. The number of trials required to obtain the equal number of fragments is time consuming and hence is to be considered for selecting the mesh concept. The concepts are also rated for pre-processing time, the development time and the complexity involved for meshing the model with the concept. As cyclic symmetry and slice geometry for meshing-hex dominant hub \& shell blades were not evaluated and the rank for patch independent (adaptive) and patch conforming (adaptive) are similar (less than uniform meshing), this concepts were not rated in Pugh matrix. The given rating for various criteria for mesh concepts are because of the following observations, considering the effect of certain criteria on mesh concept: a. Although, being simplest method of meshing, patch independent (uniform) and patch conforming (uniform) algorithm was not rated highest because for both the wheels equal number of fragments were obtained but with high mass addition due to mass scaling, giving inaccuracy in the results. b. The wheels were not meshed properly using hex dominant meshing and runtime for solution was higher compared to other concepts. c. The hex dominant mesh of the hub and tet meshing of the blades for sliced geometry for meshing do not produce equal fragments on bursting and the combination of mesh size for hub and blade is difficult to achieve. d. Pre-processing time for creating the shell blades is high and obtaining cyclic symmetric meshing demands specific analysis software for simulation. e. The development time required for slicing geometry with fragment size according to its crack propagating path is high. Moreover, it is not possible to accurately slice the wheel as the crack propagating path. f. Pattern with form part gave equal fragments for light duty and unequal fragment size for mid-range. Pattern with instance meshing gave equal number of fragments in minimumiteration, pre-processing time, development time and less complexity involved, without remarkable effect of mass scaling on accuracy of the solution. However, runtime for solution was moderately high compared to few concepts. Hence, pattern with instance meshing was ranked highest, giving this as the best concept for midrange and light duty wheel. The Pugh matrix for mesh concepts is given below and the concepts are rated on 4 point scale as: $\mathbf{0}$ - no effect, $\mathbf{1}$ - low, 3 - medium, $\mathbf{9}$ - high.

Table 1. Pugh matrix for concept selection

\begin{tabular}{|c|c|c|c|c|c|c|c|c|c|}
\hline \multicolumn{10}{|c|}{ PUGH MATRIX FOR MR AND LD WHEEL } \\
\hline Concept & Priority & $\begin{array}{c}\text { Patch } \\
\text { independent } \\
\text { (Uniform) }\end{array}$ & $\begin{array}{c}\text { Patch } \\
\text { conforming } \\
\text { (Uniform) }\end{array}$ & $\begin{array}{c}\begin{array}{c}\text { Hex dominant } \\
\text { meshing }\end{array} \\
.\end{array}$ & $\begin{array}{c}\text { Pattern meshing } \\
\text { (form part) }\end{array}$ & $\begin{array}{c}\text { Pattern meshing } \\
\text { (instance meshing) }\end{array}$ & $\begin{array}{c}\text { Slice geometry for } \\
\text { meshing (Hex hub } \\
\text { and tet blades) }\end{array}$ & $\begin{array}{c}\text { Slicing geometry } \\
\text { with fragment } \\
\text { size (Tet) }\end{array}$ & $\begin{array}{c}\text { Slicing geometry } \\
\text { with fragment } \\
\text { size (Hex) }\end{array}$ \\
\hline & & Rating & Rating & Rating & Rating & Rating & Rating & Rating & Rating \\
\hline $\begin{array}{l}\text { Number of } \\
\text { fragments }\end{array}$ & 10 & 3 & 3 & 0 & 1 & 9 & 0 & 9 & 9 \\
\hline Iterations & 8 & 3 & 1 & 0 & 1 & 9 & 1 & 0 & 0 \\
\hline \begin{tabular}{|c|} 
Mass scaling \\
effect
\end{tabular} & 10 & 1 & 1 & 0 & 9 & 9 & 0 & 0 & 0 \\
\hline \begin{tabular}{|c|}
$\begin{array}{c}\text { Run time for } \\
\text { solution }\end{array}$ \\
\end{tabular} & 6 & 3 & 3 & 0 & 1 & 1 & 1 & 9 & 9 \\
\hline $\begin{array}{c}\text { Pre-processing } \\
\text { time }\end{array}$ & 4 & 9 & 9 & 3 & 1 & 1 & 3 & 0 & 0 \\
\hline \begin{tabular}{|c|}
$\begin{array}{c}\text { Development } \\
\text { time }\end{array}$ \\
\end{tabular} & 4 & 9 & 9 & 9 & 3 & 3 & 3 & 0 & 0 \\
\hline $\begin{array}{l}\text { Complexity } \\
\text { (skills req.) }\end{array}$ & 4 & 9 & 9 & 9 & 9 & 9 & 3 & 0 & 0 \\
\hline Score & & 190 & 174 & 84 & 166 & 310 & 50 & 144 & 144 \\
\hline Rank & & 2 & 3 & 6 & 4 & 1 & 7 & 5 & 5 \\
\hline
\end{tabular}

\section{Result And Discussion}

As burst simulation with circumferential groove gave unequal number of fragments after burst, various concepts were generated and tested on midrange and light duty wheel, and the optimized concept was selected to obtain the equal number of fragments. The Explicit Dynamics was used to evaluate various concepts as it is suitable for events with high material deformation. Mass scaling effect was studied during burst simulation which can result into excess of non-physical mass addition to the smallest element, affecting the accuracy of solution due to increase in the centrifugal forces. With Patch conforming mesh and Patch independent mesh as shown in Fig.4 and Fig.5, the midrange and light duty wheels bursted with equal number of fragments but even 
at the velocity less than the specified target burst speed, giving unrealistic burst scenario, due to excess of mass added at the blades of wheel due to mass scaling. In hex dominant meshing, the pyramids are splitted into tet elements, further reducing the element size. Proper meshing was possible only if wheels are fine meshed, resulting in increase in the runtime for solution and the mass added due to mass scaling. The pattern with instance meshing shown in Fig.6 gave equal number of nodes and elements in each sectors and pre-processing time was less due to parallel meshing. The wheel bursted with equal fragments and mass added was high with no significant effect on the accuracy of the solution. For Sliced-Hex dominant hub and Tet blades, while the light duty wheel was not meshed properly, wheel bursted with unequal number of fragments for mid-range wheel and the combination of the hub and blades mesh size was not possible after certain combinations of mesh sizes for meshing the wheel. Fragment with tet elements bursted in minimum runtime but the development time required for Slice geometry with fragment size is very high as slicing would require a proper observation for crack propagating path and slicing the wheel accordingly which is an intense job, with uncertainty of the sliced blade being similar to the fragment size.

The Pugh selection method shown in Table 1 was used to select the concept by rating the concepts for various criteria and pattern-instance meshing was selected as the best concept for meshing among the evaluated concepts. The Patch independent with uniform meshing was rated second highest and could be used as an alternative mesh concept but with proper mesh size that will not affect the burst simulation due to mass scaling. The burst simulation for different mid-range and light duty wheels gave equal fragments with pattern-instance meshing.

\section{Conclusion}

The burst simulation of turbine wheel for containment test, with circumferential groove as a weakening slot yields unequal number of fragments after burst even when the model is cyclic symmetry. One of the major reasons for the randomness in the fragment sizes is due to meshing of the model.

1. Light duty and midrange wheels were evaluated with different meshing algorithms and mesh concepts. The variation in the number of fragments was observed with different mesh concepts, justifying the mesh as a basis for randomness in fragment size.

2. The concepts were rated for different criterion and highest rated concept was selected using Pugh selection method. The pattern with instance meshing was selected as a best concept for meshing the model and was rated highest in Pugh matrix, as it is observed to give equal number of fragments in less runtime without affecting the accuracy of solution due to mass scaling. The burst simulation for different mid-range and light duty wheels gave equal fragments with pattern-instance meshing.

3. The pattern with instance meshing needs cyclic symmetric base model and hence, with unavailability of sector, patch independent algorithm with uniform meshing can be used as an alternative, but with proper judgement of mass scaling that can affect the accuracy of solution.

4. Even though the concept is not applicable for the straight grooves, as a future work, the concept can be evaluated for the wheels with angular grooves.

\section{References}

[1] White paper No.2, Burst \& containment: Ensuring Turbocharger safety, Garret by Honeywell.

[2] J M Ramamoorthy, S S Parikh, S Pandian, P S Kasthuri Rangan , Containment simulation and validation of turbocharger housing design, 11th International Conference on Turbochargers and Turbocharging, London (2014).

[3] L. Wang, D. M. Eastwood, Explicit dynamic finite element simulation of turbocharger containment and wheel burst, Cummins Turbo technologies, UK.

[4] G.R. Johnson, W.H. Cook, A Constitutive Model and Data for Metals Subjected to Large Strains, High Strain Rates and High Temperatures, Proceedings of the Seventh International Symposium on Ballistics, Hague, the Netherlands (1983).

[5] Xuan Hai-jun, Wu Rong-ren, Aeroengine turbine blade containment tests using high-speed rotor spin testing facility, Aerospace Science and Technology 10 ,2006, 501-508.

[6] Hai-jun XUAN, Lu-lu LIU, Yi-ming FENG, Qing HE, Juan-juan Li.,Containment of high speed rotating disk, Journal of Zhejiang University-SCIENCE A (Applied Physics \& Engineering,13(9), 2012,665-673.. 